\title{
A Recent Study on Routing Protocols in UWSNs
}

\author{
Muhammad Ahsan \\ Department of Computer Science, \\ Preston University, \\ Peshawar, Pakistan \\ Sheeraz Ahmed \\ Faculty of Engineering and \\ Technology, \\ Gomal University, \\ Dera Ismail Khan, Pakistan
}

\author{
Adil khan \\ Department of Computer Science, \\ Abdul Wali Khan University, \\ Mardan, Pakistan \\ Mukhtaj khan \\ Department of Computer Science, \\ Abdul Wali Khan University, \\ Mardan, Pakistan
}

\author{
Fazle Hadi \\ Department of Computer Science, \\ Preston University, \\ Peshawar, Pakistan \\ Fazal Wahab \\ Department of Electrical \\ Engineering, \\ University of Engineering and \\ Technology, \\ Peshawar, Pakistan
}

\author{
Imran Ahmed \\ Department of Computer Science, \\ Institute of Management Sciences, \\ Peshawar, Pakistan
}

\begin{abstract}
Recent research has seen remarkable advancement in the field of Under Water Sensor Networks (UWSNs). Many different protocols are developed in the recent years in this domain. As these protocols can be categorized in a variety of ways according to the mechanisms and functionalities they follow, hence it becomes important to understand their principal working. In this research we have introduced three analysis methods; Clustering based, Localization based and Cooperation based routing by selecting some recent routing protocols in the field of UWSN and presented a comparative analysis according to the categories in which they lie. This research has been taken theoretically and is qualitative one. Also a detail analysis of their key advantages and flaws are also identified in this research.
\end{abstract}

Keywords-UWSN; routing protocol; relay node; sink

\section{INTRODUCTION}

In the past decades researchers have shown a lot of interest in the field of UWSNs, because of its wide range of different applications. UWSNs is actually a sub area of Wireless Sensor Networks (WSNs), which facilitate on radio communication with high-precision of information to a sink node from any of the source node. UWSN is a representation of three dimensional information as like marine rescuing and tracking of submarine and exploration of oceans [1].

UWSN is being used for several applications such as environmental monitoring, reconnaissance data acquisition and tactical surveillance. The sensors are interconnecting with wireless links which further propagate optical and radio signals using acoustic signals affected by heavy amount of scattered and absorption loss. These acoustic signals are imposed by different challenges on communication protocol like high error bit rate, limiting bandwidth and delay in long propagation [2]. UWSNs consist of underwater acoustic sensor nodes. In addition to compare with new approaches to ocean-column and ocean-bottom monitoring, UWSNs are used for collecting real time information gathering from a specific area which increase the efficiency of applications like warship monitoring for real time, oceanic graphical data collection, offshore exploration, disaster prevention and environmental monitoring [3].

Each sensor is attached with acoustic modem for low bandwidth and an antenna [4]. UWSN consisting of number of sensor nodes have $\operatorname{sink}(\mathrm{s})$ placed at the surface of the water. Here the transmission of data can be obtained by different parameters such as optical, radio or sound waves. Light waves and frequency are not as much suitable due to the high absorption, attenuation and scattering as there is very large area for monitoring in sea so distance transmission creates problems [5]. UWSNs are used for the monitoring requirement of aqueous environment where the acoustic sensors gather interesting information and forward this data to the end node using any specific routing technique [6]. Figure 1 shows the general phenomena of UWSNs. 


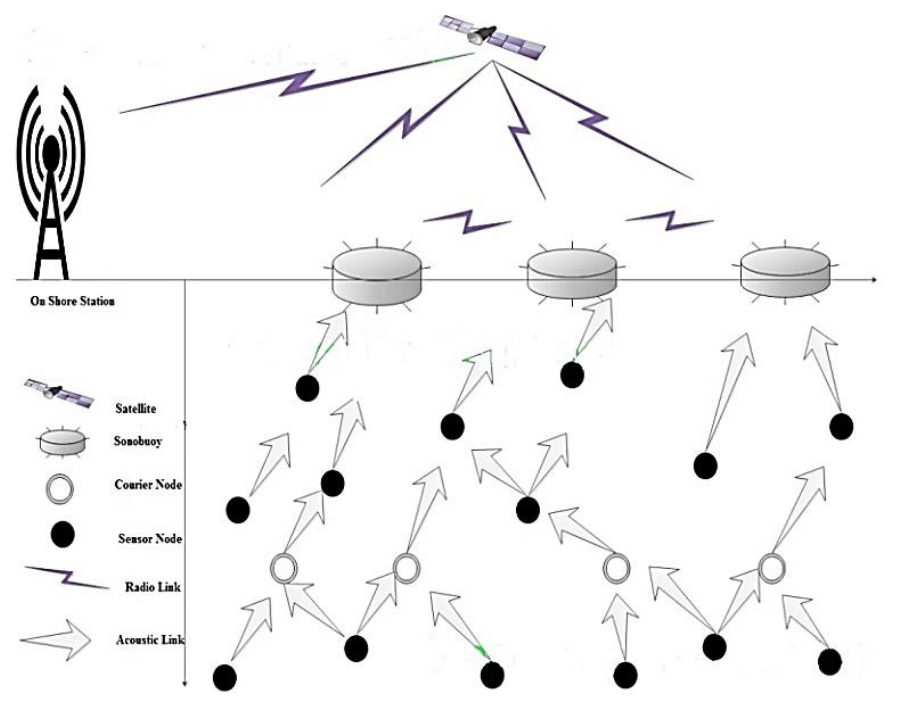

Fig. 1. Underwater Sensor Network Architecture

Communication among signals is not feasible due to the absorption of high radio signals and rapid attenuation in UWSNs. Hence, low absorption rate is required at that stage where the acoustic signals are used much in a wide range and due to these reasons such as end-to-end delay of signals and low bandwidth, the routing mechanism becomes a very major issue or challenge in UWSNs [7]. UWSN performs monitoring task in collaboration over a specified are consisting of vehicles and sensors. For the high data rate of transmission in underwater communication, acoustic waves are the most desirable source [8]. UWSN is a study of art approaches to explore potential information. Underwater communication has different specifications like low bandwidth capacity, mobility, propagation delay, memory and battery limit. Recent researches for investigating the underwater behavior were based on territorial technology of UWSNs. As water is used as medium of transmission and the required signals to transmit data so UWSNs shows different structure design as comparison to terrestrial network. In USWNs different schemes have been proposed for efficient and cooperative routing having transmission with usage of sink followed by relay/nodes [9]. The node mobility changes the structure of topology in underwater environment with respect to water current and water pressure. Dynamic node movement has also been proposed by several authors. The existing problems in mobility of nodes has already discussed in [23].

\section{Routing PROTOCOLS}

Many researchers have proposed different techniques and protocols to achieve performance and stability of network. Some of them recently introduced are summarized and discussed below as a comparative study. All the below mentioned protocols are divided into 3 major categories:

1) Clustering-based routing

a) Round-Based Clustering

b) $S E E C$

c) $E B E C R P$
2) Localization-free routing

a) $E E-D B R / D-D B R$

b) $B T M$

c) $i A M C T D$

d) $A E D G$

3) Cooperation-based routing

a) $\mathrm{Co}-U W S N$

b) $C o-D B R$

c) $S P A R C O$

d) $D E A D S$

\section{Clustering-BAsed Routing}

\section{A. Round-Based Clustering}

A Round Based Clustering Scheme is proposed where redundant data transmission has been resolved in the network as this scheme offers reduced network lifetime. The proposed scheme works in rounds, which consisted of four phases that are: data aggregation, clustering, cluster-head and initialization. The proposed scheme increased network throughput as well as give guarantee of sink to receive minimum percentage of data at the base station, demonstrated by simulation results. The cluster heads were chosen by a selfselection process which was based on distance to the base station and residual energy [10].

\section{B. SEEC}

Regarding efficient energy, many routing protocols are proposed in UWSNs. A health protocol named Sparsity-aware Energy Efficient Clustering (SEEC) is introduced which specifically search sparse regions of network. In SEEC with the assist of Density Search Algorithm and Sparsity Search Algorithm, the region is divided into equal size of sub regions and search dense and sparse regions of the network field. SEEC achieved stability of network in dense region of the network with optimal number of cluster where a static cluster is represented by each dense region [11].

\section{EBECRP}

In UWSN because of harsh environment of underwater battery replacement is an expensive task such as where nodes attach with limited battery power. Therefore a Balanced Energy and an energy Efficient consumption Cluster Based Routing Protocol (EBECRP) has been introduced in UWSN. In depth based routing generally the nodes lying near the sink nodes die early because of unbalancing load over node near sink, so EBECRP avoids this depth based routing and balancing load over all nodes using mobile sinks. For maximum energy consumption this protocol used clustering concept for reducing multi-hop where cluster head being selected for collecting data from one neighbor node into locally compressed communication for reducing global communication. For reducing number of transmission clustering based technique is used as here which frequently changes the position of sink using mobile sink nodes. EBECRP have achieved several parameters like network life time and having maximum stability period [12]. 


\section{LOCALIZATION-FrEe ROUTING}

\section{A. $E E D B R / D-D B R$}

As depth-based routing protocol (DBR) [13] uses depth information only which is more practical for UWSNs. However, depth information is not enough to restrict packets to be forwarded within a particular area. Packets may be forwarded through multiple paths which might affect energy wasted and increased end-to-end delay. In this paper, the authors introduced underwater Time Of Arrival (ToA) ranging technique to address the problem. To maintain all the original advantages of DBR, they made the following contributions: Energy-Efficient Depth-Based Routing protocol EE-DBR which decreased the redundancy of cost energy in some blind zones; low-latency depth-based routing protocol D-DBR which is able to deliver a packet via an optimal path. The EEDBR/D-DBR is found more successful by simulations up to $90 \%$ when greater than 400 numbers of nodes are used. As EEDBR/D-DBR having greater successive delivery ratio in dense network so it dramatically consumed energy. Everywhere EE-DBR will be the better choice wherever energy is considered as a target parameter like data sampling of long-time marine hence also providing better performance in the same way [14].

By using ranging technique of ToA distance is measured by propagation between two nodes. EEDBR aims to decrease redundancy forwarding of multipath and considered the following equations

$$
\left(\operatorname{dis}^{2}-\Delta d^{2}\right)+(R-\Delta d)^{2}>R 2
$$

where dis is distance between node $\mathrm{A}$ and sensor node $\mathrm{S}$, depth difference is denoted by $\Delta \mathrm{d}$ and maximum transmission by $\mathrm{R}$, node is able to figure out $\Delta \mathrm{d}$ and dis while receiving packet, so EE-DBR in build zone eliminates redundancy cost energy of sensors and D-DBR directed packets to sink through an optimal path and reduced propagation delay and hop numbers in the scenario of single sink, following equation is considered

$$
F(d, \theta)=\omega_{1} f(d)+\omega_{2} g(\theta) \quad \theta \in[0, \Pi]
$$

Where, $g(\theta)$ is defined as angle holding time, $f(d)$ as sum of depth holding time, in this way author implemented directional depth-based routing, while sink node float on surface [14].

\section{B. BTM}

Balance Transmission Mechanism (BTM) elaborates direct acoustic transmission when in multi-hop transmission consumption of local excessive energy is required. BTM [15] chooses single hop or multi-hop transmission of mode to thesink on the same energy level of underwater sensor networks. Different hop by hop schemes have been introduced for a multi-hope UWSN where data packets forwarded by downstream nodes works as ACKs for the previously transmitted data packets. Another scalable and for providing time efficient routing a protocol is been introduced as a dynamic addresses based hop-by-hop routing protocol for UWSN [15].
For broadcasting backwards the EL notice packet (ELNP), while network collapse; the maximum energy consumption is defined as

$$
E_{b b}=\frac{\sum_{i=2}^{N}(2 \mathrm{i}-1) P_{0} r^{k} a^{r}}{n}
$$

where the number of the nodes is $(2 i-1)$ in Slice $i$, total maximum time of broadcasting backward ELNPs in slice is given as $(2 i-1) / n$ and in Slice $i$ average distance of sending backward the ELNP is $r$.

In slice the remaining maximum energy of 1 node can be determined as $(i-1) \mathrm{E} 0 / m$, while network collapse, then remaining pessimistic energy of total nodes is determined as

$$
E_{p r}=\frac{\sum_{i=2}^{N}(2 \mathrm{i}-1)(i-1) E_{0}}{m}
$$

The wasted maximum energy in this scheme is dissipated as

$$
E w=E b b+E p r
$$

This algorithm introduces efficient routing algorithm in the phase of route-set-up, where routing algorithm near the optimum transmission selected a relay node. Balance transmission algorithm balanced energy consumption. By the current EL of successor nodes the underwater sensor nodes decides their transmission mode. To be balance the energy consumption for better energy consumption optimal classification number of EL is being evaluated [15].

\section{C. $i A M C T D$}

AMCTD [16] is an earlier protocol which maximizes the network life time due to exploits the adaptive mobility of courier nodes. AMCTD'S performance is far better than EEDBR and DBR, however it had deficiency which further removed by an Improved Adaptive Mobility of Courier Nodes in Threshold-Optimized DBR Protocol (iAMCTD) which is routing protocol overcome to AMCTD uses forwarded function for time critical applications for UWSN [17]. iAMCTD exploits network density to tackle the problem of path loss, flooding and latency propagation. In sparse condition the energy consumption is decreases of low depth nodes and minimizes end-to-end delay due to courier nodes of efficient node [17]. In terms of distance and frequency the acoustic link channel is described in iAMCTD, faced transmission of redundant data which further resulted in major energy consumption

\section{D. $A E D G$}

Some existing proposed schemes collected data from the Member Nodes (MNs) and transmitting this to Mobile Sink (MS) using GNs, where nodes of Received Signal Strength Indicator (RSSI) values been selected as Gateway Nodes (GNs) . Authors in [18] described several factors which affects in the underwater communication environment. Routing task is more challenging in underwater environment as compared to terrestrial WSNs hence An Efficient Data Gathering 
(AEDG) as a new routing protocol been introduced in UWSNs for the reliable data delivery of data. AEDG implies Autonomous Underwater Vehicle for collecting data from gateways while associating sensor nodes with the gateway to prolong network lifetime and used Shortest Path Tree (SPT) algorithm [18].

\section{COOPERATION-BASED ROUTING}

\section{A. Co-UWSN}

Cooperative routing protocol involves sink node or relay node for data transmission. In Co-UWSN due to cooperation at node level, the energy consumption is minimized and throughput becomes high. The main purpose of Co-UWSN the cooperative routing protocol between nodes is utilized in data transmission of more than one node. In Co-UWSN, a Cooperation Based mechanism is introduced having minimum path loss over link, single and multi-hop merits were utilized for routing data via underwater networks. Here a cost function has mentioned to select the very best route to sink calculated on the basis of their residential energy and their distance from the sink. Co-UWSN reduces effects of path loss and enhanced stability of the network [19].

In Co-UWSN, Depth of sensors are updated and informed to neighbors and when sink broadcast a packet of information containing node ID, depth and energy status, sink sends a help message to all nodes to have the vital information of all nodes. By employing a hello packet by each node is identified by its neighbor in the transmission range while maintain a separate queue under depth threshold of neighbors for identifying finest forwarder to transmit data and Weight is calculated by each node by the given formula

$$
\mathrm{W}_{\mathrm{i}}=\frac{\left(\max \left(p\left(d_{S 1 R 1}, f\right), p\left(d_{S 1 D 1}, f\right)\right)+\max \left(R E_{R 1} R E_{D 1}\right)\right.}{\min \left|d_{S 1 R 1}\right| 2,\left|d_{S 1 D 1}\right| 2}
$$

\section{B. $C o D B R$}

In order to have reliability and efficient throughput a Cooperative Depth Base Routing (Co-DBR) is introduced for UWSN which employs cooperation in DBR. Cooperative transmission technique is introducing physical layer aspects of Under Water Acoustic Communication (UWAC) which further demonstrate the point-to-point counterparts for superiority of cooperative UWAC. The Underwater Decode and Forward and Underwater Amplify and Forward are two asynchronous cooperative data transmission techniques used for improving performance of network [20]. Co-DBR showed 98\% more packet acceptance ratio, 90\% less packet drop and $83 \%$ more throughput in stable region as compare to noncooperative schemes. Because of its less packet drop CoDBR is proved to be more beneficial for critical mission applications [20].

\section{SPARCO}

Recently Stochastic Performance Analysis with Reliability and Cooperation (SPARCO) is cooperation based routing protocol for UWNs for enhancing the performance, SPARCO came in UWSNs for cooperative communication of energy efficiency routing schemes. In SPARCO, an approach is suggested with much low path loss to forward information linking with feature using single and multi-hop in addition the proposed protocol used a cost function for finding most suitable path for sink. The outage probability is totally dependent on the depth of the ocean at any instant and noise factors and attenuation occurring in the ocean currents. In terms of SNR, it can be written is [21]

$$
\mathrm{P}_{\text {outage }}=\mathrm{P}\left\{\hat{P}(t)<2 \pi H \exp \left(\frac{2^{\left(\frac{R}{B}\right)}+S L+p(d, f)}{20}\right)\right\}
$$

where $\mathrm{H}$ is depth of water and $(\mathrm{d}, \mathrm{f})$ is denoted as distance and frequency, SPARCO solves problems of Attenuation, bandwidth, noise and reliability.

\section{DEADS}

Many unintended sensor nodes transmit signals to be overheard in the broadcast nature of wireless communication which is an advantage of cooperative communication. An alternative mechanism been proposed to minimize the effect of fading over multi-hop communication. Many protocols have been proposed so far for the cooperative communication in UWSNs to improve the system performance including cooperation at MAC layer. A new scheme further developed using two relay nodes either Multiple (DEADS-MRC) or Single Relay Communication (DEADS-SRC) for some enhancement of UWSNs to maximize throughput, to minimize Bit Error Rate and to conserve energy however sink mobility is not been yet considered as cooperative routing, hence DEADS aim is to bring efficiency due to sink mobility, dominating set based cooperative routing and to have network reliability [22].

\section{CONCLUSION \& FUTURE WORK}

In this paper different surveys of different routing protocols have been conducted for the new researchers working in the area of UWSNs. Many protocols are presented for energy efficiency, time efficiency and stability of network to maximize throughput and to minimize the delay in forwarding data to the sink node which are further mentioned in Table 1. Routing protocols employing depth based routing, localization based, cooperation based or clustering based all of these techniques have been defined. We have mentioned different advantages and flaws of these routing protocols introduced recently for data transmission in UWSNs. Some protocols that are recently produced reduce energy consumption and enhance network stability of underwater networks but the research continues to work on high energy consumption and higher throughput; reduced transmission loss, processing overhead and redundant data transmissions.

\section{REFERENCES}

[1] Akyildiz, Ian F., Dario Pompili, and Tommaso Melodia. "Underwater acoustic sensor networks: research challenges." Ad hoc networks 3, no. 3 (2005): 257-279.

[2] Javaid, Nadeem, Mehreen Shah, Ashfaq Ahmad, Muhammad Imran, Majid Iqbal Khan, and Athanasios V. Vasilakos. "An Enhanced Energy Balanced Data Transmission Protocol for Underwater Acoustic Sensor Networks." Sensors 16, no. 4 (2016): 487.

[3] Jiang, Jinfang, Guangjie Han, HuiGuo, Lei Shu, and Joel JPC Rodrigues. "Geographic multipath routing based on geospatial division in duty-cycled underwater wireless sensor networks." Journal of Network and Computer Applications 59 (2016): 4-13. 
[4] Lee, Uichin, Paul Wang, Youngtae Noh, Luiz Filipe M. Vieira, Mario Gerla, and Jun-Hong Cui. "Pressure Routing for Underwater Sensor Networks." In INFOCOM, pp. 1676-1684. 2010.

[5] ur Rahman, Zia, Zaheer Ahmad, Amir Murad, Tanveer Khan, Zahoor Ali Khan, Umar Qasim, and Nadeem Javaid. "On utilizing static courier nodes to achieve energy efficiency with depth based routing for underwater wireless sensor networks." In 2016 IEEE 30th International Conference on Advanced Information Networking and Applications (AINA), pp. 1184-1191. IEEE, 2016.

[6] Guo, Xiaoxing, Michael R. Frater, and Michael J. Ryan. "A propagation-delay-tolerant collision avoidance protocol for underwater acoustic sensor networks." In OCEANS 2006-Asia Pacific, pp. 1-6. IEEE, 2007.

[7] Zidi, Chaima, Fatma Bou abdallah, and Raouf Boutaba. "Routing design avoiding energy holes in underwater acoustic sensor networks." Wireless Communications and Mobile Computing (2016).

[8] Pompili, Dario, and Ian F. Akyildiz."Overview of networking protocols for underwater wireless communications." IEEE Communications Magazine 47, no. 1 (2009): 97-102.

[9] Liaqat, Tayyaba, Nadeem Javaid, Syed Muaraf Ali, Muhammad Imran, and Mohammed Alnuem. "Depth-Based Energy-Balanced Hybrid Routing Protocol for Underwater WSNs." In Intelligent Networking and Collaborative Systems (INCOS), 2015 International Conference on, pp. 262-267. IEEE, 2015.

[10] Tran, Khoa Thi-Minh, and Seung-Hyun Oh. "Uwsns: A round-based clustering scheme for data redundancy resolve." International Journal of Distributed Sensor Networks 2014 (2014).

[11] Azam, Irfan, Abdul Majid, Ijaz Ahmad, Usman Shakeel, Hamad Maqsood, Zahoor Ali Khan, Umar Qasim, and Nadeem Javaid. "SEEC: Sparsity-aware energy efficient clustering protocol for underwater wireless sensor networks." In 2016 IEEE 30th International Conference on Advanced Information Networking and Applications (AINA), pp. 352-361. IEEE, 2016.

[12] Majid, Abdul, Irfan Azam, Abdul Waheed, Muhammad Zain-ul-Abidin, Taimur Hafeez, Zahoor Ali Khan, Umar Qasim, and Nadeem Javaid. "An energy efficient and balanced energy consumption cluster based routing protocol for underwater wireless sensor networks." In 2016 IEEE 30th International Conference on Advanced Information Networking and Applications (AINA), pp. 324-333. IEEE, 2016.

[13] Yan, Hai, Zhijie Jerry Shi, and Jun-Hong Cui. "DBR: depth-based routing for underwater sensor networks." In International Conference on Research in Networking, pp. 72-86. Springer Berlin Heidelberg, 2008.

[14] Diao, Boyu, YongjunXu, ZhulinAn, Fei Wang, and Chao Li. "Improving both energy and time efficiency of depth-based routing for underwater sensor networks." International Journal of Distributed Sensor Networks 2015 (2015): 8.

[15] Cao, Jiabao, Jinfeng Dou, and Shunle Dong. "Balance transmission mechanism in underwater acoustic sensor networks." International Journal of Distributed Sensor Networks 2015 (2015): 2.

[16] Jafri, MohsinRaza, Shehab Ahmed, NadeemJavaid, Zainal Ahmad, and R. J. Qureshi. "Amctd: Adaptive mobility of courier nodes in thresholdoptimized dbr protocol for underwater wireless sensor networks." In Broadband and Wireless Computing, Communication and Applications (BWCCA), 2013 Eighth International Conference on, pp. 93-99. IEEE, 2013

[17] Javaid, Nadeem, Mohsin Raza Jafri, Zahoor Ali Khan, Umar Qasim, Turki Ali Alghamdi, and Muhammad Ali. "Iamctd: Improved adaptive mobility of courier nodes in threshold-optimized dbr protocol for underwater wireless sensor networks." International Journal of Distributed Sensor Networks 2014 (2014): 1.

[18] Javaid, Nadeem, Naveed Ilyas, Ashfaq Ahmad, Nabil Alrajeh, Umar Qasim, Zahoor Ali Khan, Tayyaba Liaqat, and Majid Iqbal Khan. "An Efficient Data-Gathering Routing Protocol for Underwater Wireless Sensor Networks." Sensors 15, no. 11 (2015): 29149-29181.

[19] Ahmed, S., N. Javaid, F. A. Khan, M. Y. Durrani, A. Ali, A. Shaukat, M. M. Sandhu, Z. A. Khan, and U. Qasim. "Co-UWSN: cooperative energy-efficient protocol for underwater WSNs." International Journal of Distributed Sensor Networks 2015 (2015): 75.

[20] Nasir, Hina, Nadeem Javaid, H. Ashraf, S. Manzoor, Zahoor Ali Khan, Umar Qasim, and M. Sher. "CoDBR: cooperative depth based routing for underwater wireless sensor networks." In Broadband and Wireless Computing, Communication and Applications (BWCCA), 2014 Ninth International Conference on, pp. 52-57. IEEE, 2014.

[21] Ahmed, Sheeraz, Nadeem Javaid, Ashfaq Ahmad, Imran Ahmed, Mehr Yahya Durrani, Armughan Ali, Syed Bilal Haider, and Manzoor Ilahi. "SPARCO: Stochastic Performance Analysis with Reliability and Cooperation for Underwater Wireless Sensor Networks." Journal of Sensors 2016 (2016)

[22] Umar, Amara, Nadeem Javaid, Ashfaq Ahmad, Zahoor Ali Khan, Umar Qasim, Nabil Alrajeh, and Amir Hayat. "DEADS: Depth and Energy Aware Dominating Set Based Algorithm for Cooperative Routing along with Sink Mobility in Underwater WSNs." Sensors 15, no. 6 (2015): 14458-14486.

[23] Ahmed, Mukhtiar, Mazleena Salleh, and M. Ibrahim Channa. "Routing protocols based on node mobility for Underwater Wireless Sensor Network (UWSN): A survey." Journal of Network and Computer Applications 78 (2017): 242-252. 
TABLE I. COMPARISON OF UWSN ROUTING PROTOCOLS

\begin{tabular}{|c|c|c|c|}
\hline Protocol Name & Key Parameters & Achievements & Disadvantages/ Flaws \\
\hline EEDBR/D-DBR [14] & $\begin{array}{l}\text { - } \quad \text { Minimize energy consumption } \\
\text { - } \quad \text { Minimum end to end delay }\end{array}$ & $\begin{array}{ll}\text { - } & \text { Minimize Energy Consumption } \\
\text { - } & \text { Delivery Ratios } \\
\text { - } & \text { End-to-End Latency } \\
\end{array}$ & $\begin{array}{l}\text { Collision due to Redundant } \\
\text { Data transmission }\end{array}$ \\
\hline $\begin{array}{l}\text { Round-Based } \\
\text { clustering [10] }\end{array}$ & $\begin{array}{ll}- & \text { Residual energy nodes } \\
\text { - } & \text { Clustering based routing }\end{array}$ & $\begin{array}{ll}- & \text { Energy consumption } \\
- & \text { Network life time improved } \\
- & \text { Throughput and } \\
\end{array}$ & $\begin{array}{l}\text { - } \quad \text { Computation overhead } \\
\text { - } \quad \text { Transmission delay }\end{array}$ \\
\hline BTM [15] & $\begin{array}{ll}\text { - } & \text { Energy Consumption } \\
\text { - } & \text { Path Loss and Bandwidth }\end{array}$ & $\begin{array}{ll} & \text { Network Life Time } \\
\text { - } & \text { Balanced energy consumption }\end{array}$ & - Transmission delay \\
\hline Co-UWSN [19] & $\begin{array}{ll}- & \text { Depth based } \\
\text { - } & \text { Multi-hop Cooperation }\end{array}$ & $\begin{array}{ll} & \text { Minimum transmission loss } \\
\text { - } & \text { Network lifetime and high } \\
\text { stability }\end{array}$ & - Increased end to end delay \\
\hline Co-DBR [20] & $\begin{array}{l}-\quad \text { Depth based routing with } \\
\text { - } \quad \text { Cooperation, Localization }\end{array}$ & $\begin{array}{ll}- & \text { Network reliability and } \\
\text { throughput efficiency } \\
\text { - } & \text { Reduced packet drop } \\
\end{array}$ & $\begin{array}{l}\text { - } \quad \text { Greater end-to-end delay } \\
\text { - Unbalanced energy } \\
\text { consumption }\end{array}$ \\
\hline iAMCTD [17] & $\begin{array}{ll}- & \text { Network Density Link Status } \\
\text { - } & \text { Residual Energy Depth } \\
\text { - } & \text { Reduce transmission loss }\end{array}$ & $\begin{array}{ll}- & \text { High energy efficiency } \\
- & \text { High stability period } \\
\bullet & \text { Minimize end-to-end packet } \\
\text { - } & \text { delay } \\
\end{array}$ & $\begin{array}{l}\text { - } \quad \text { Minimum throughput } \\
\text { - } \quad \text { Control packets over headed }\end{array}$ \\
\hline AEDG [18] & $\begin{array}{l}\text { - } \quad \text { Data gathering with multi } \\
\text { gateways } \\
\text { - } \quad \text { Routing query and feedback } \\
\text { from sink }\end{array}$ & $\begin{array}{ll}\text { - } & \text { High energy efficiency } \\
\text { - } & \text { High stability period } \\
\text { - } & \text { High network throughput } \\
\end{array}$ & $\begin{array}{ll}- & \text { High end-to-end delay } \\
- & \text { Minimum throughput } \\
\text { - } & \text { Increase energy } \\
& \text { consumption } \\
\end{array}$ \\
\hline SPARCO [21] & $\begin{array}{l}\text { - } \quad \text { Signal to Noise Ratio } \\
\text { - } \quad \text { Path Selection }\end{array}$ & $\begin{array}{ll}- & \text { Enhanced stability period } \\
\text { - } & \text { Increase network life time } \\
\text { - } & \text { Throughput increases } \\
\end{array}$ & - $\quad$ Energy Consumption \\
\hline SEEC [11] & $\begin{array}{l}\text { - } \quad \text { Clustering based routing } \\
\text { - } \quad \text { Sparse regions of equal size }\end{array}$ & $\begin{array}{ll}- & \text { Minimizes network energy } \\
\text { - } & \text { Consumption with balanced } \\
\text { scheme }\end{array}$ & - $\quad$ Minimum throughput \\
\hline DEADS [22] & - Depth residual energy & $\begin{array}{ll}\text { - } & \text { Network reliability and Network } \\
\text { efficiency } \\
\text { - } & \text { Increased throughput } \\
\text { - } & \text { Reduced packet drop } \\
\end{array}$ & $\begin{array}{ll}- & \text { Processing Overhead } \\
\text { - } & \text { Short stability period } \\
\text { - } & \text { High energy consumption } \\
\text { - } & \text { Short stability period } \\
\end{array}$ \\
\hline EBECRP [12] & - Clustering based routing & $\begin{array}{l}\text { - } \quad \text { Maximum throughput } \\
\text { - } \quad \text { Maximum energy consumption }\end{array}$ & $\begin{array}{l}\text { - } \quad \text { Computation due to cluster } \\
\text { head selection }\end{array}$ \\
\hline
\end{tabular}

